\section{Estimation of haemoglobin using a Technicon AutoAnalyzer}

\section{J. H. KEMP From the Department of Pathology, Grimsby General Hospital, Grimsby}

This paper describes a method for the routine estimation of haemoglobin, after conversion to cyanmethaemoglobin, using the AutoAnalyzer. Details are given of the preparation of a stable red cell suspension, and of the method of calibration of the AutoAnalyzer, using this suspension, which allows for the variation in sample dilution due to normal wear and tear of the pump tubes. The manifold (Fig. 1) reduces error due to carry-over and allows full conversion to cyanmethaemoglobin with the potassium ferricyanide solution.

\section{REAGENTS}

POTASSIUM FERRICYANIDE-CYANIDE SOLUTION Sodium bicarbonate, $50 \mathrm{~g}$., potassium ferricyanide, $10 \mathrm{~g}$., and potassium cyanide, $2 \cdot 5 \mathrm{~g}$., are dissolved in distilled water and made up to 1 litre. To $40 \mathrm{ml}$. of this stock solution are added Brij $350.5 \mathrm{ml}$., ammonia solution $0.8800 .4 \mathrm{ml}$., and the volume made up to 1 litre with distilled water.

RED CELL SUSPENSION Blood is taken into acid-citratedextrose solution in the proportion $3: 1$ and allowed to Received for publication 17 January 1966. stand at $4^{\circ} \mathrm{C}$. until packed. The plasma is aspirated $15 \mathrm{ml}$. Streptolin 33 (Glaxo) added to the cells which afe run through a fine nylon filter to remove any clots. Frow this stock suspension $80 \%, 60 \%$, and $40 \%$ dilutiof $(100 \mathrm{ml}$. each) are prepared using a sterile saline $(100 \mathrm{mp})$ and Streptolin $33(5 \mathrm{ml}$.) mixture as diluent. The stod suspension and its dilutions are stored at $4^{\circ} \mathrm{C}$. in $4 \mathrm{~m} \overline{\mathrm{p}}$ quantities in sterile Bijou bottles and have a usable lifie in excess of three months. These are used in the day-to day calibration of the AutoAnalyzer.

\section{STANDARDIZATION OF SUSPENSIONS}

Dilution and conversion of the red cell suspensions cyanmethaemoglobin followed by comparison with Cyanmethaemoglobin standard (B.D.H.) in a filter phot $\oplus$ meter proved unsatisfactory as a method of standardization. The haemoglobin values of subsequent bloo samples, when diluted manually and read in a filter photometer, differed from those obtained when using the Aut $\&$ Analyzer by some $7 \%$. This variation is thought to be due to differences in the spectral characteristics of the twe types of filter and, possibly, to the methods of recording even though Beer's law was obeyed using both instry ments. As it is essential that strictly comparable resulg should be obtained irrespective of the instrument used an indirect method of standardization of the red ceff suspensions was devised.

The haemoglobin values of 10 blood samples in the range $10-14 \mathrm{~g} . / 100 \mathrm{ml}$. are determined accurately with

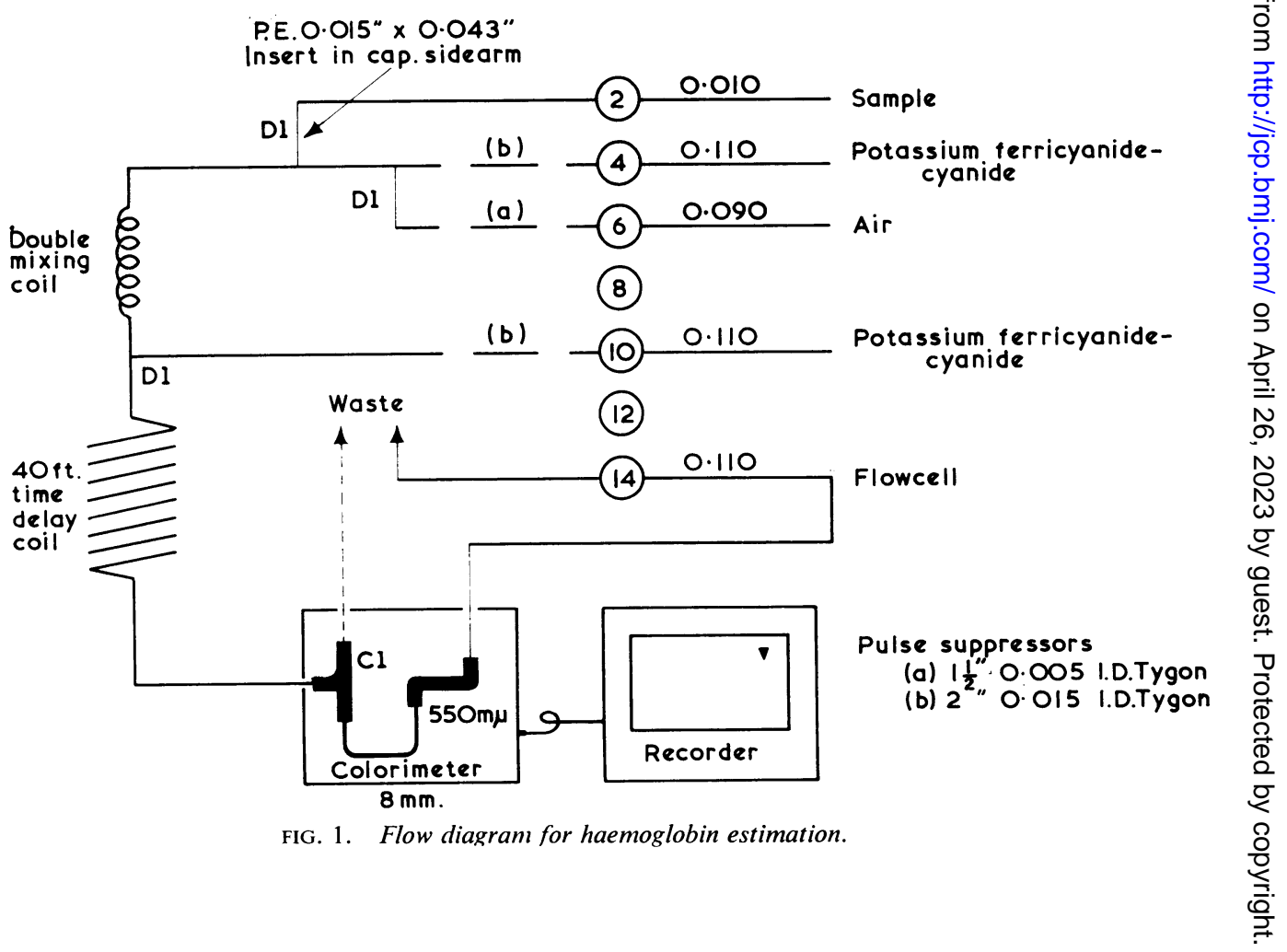




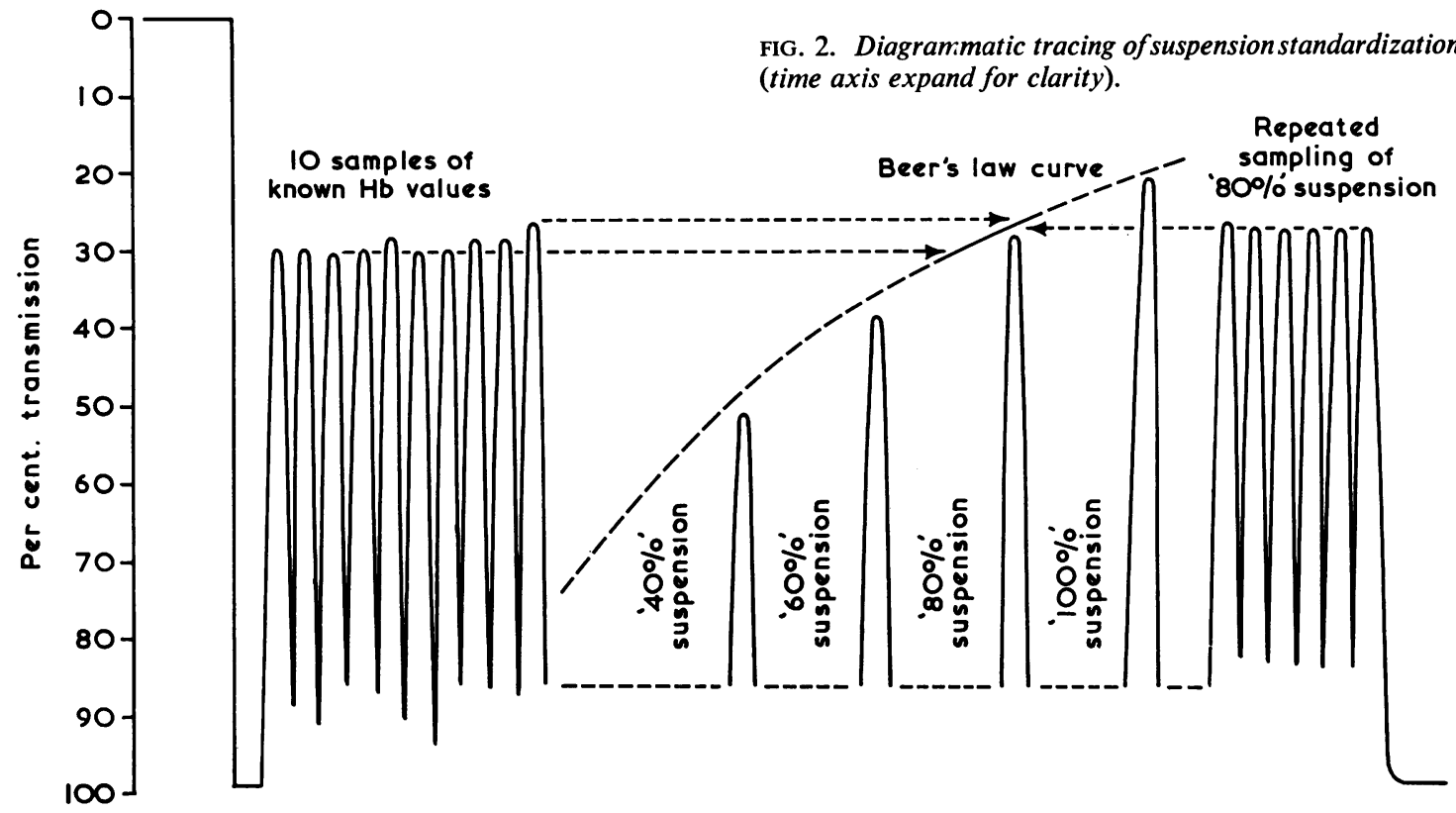

FIG. 2. Diagrammatic tracing of suspension standardization (time axis expand for clarity).

filter photometer. After establishing $0 \%$ and $99 \%$ transmission lines on the AutoAnalyzer these samples are run at $60 / \mathrm{hr}$. followed by the red cell suspensions in order of increasing concentration, these in turn being followed by repeated sampling of the suspension which comes within the range $10-14 \mathrm{~g} . / 100 \mathrm{ml}$. (Fig. 2). Adequate mixing of the sample is effected by the use of a Technicon VibraMixer accessory attached to the crook of the MK. I sampler. The peak heights of the blood samples are plotted against their determined haemoglobin values using the AutoAnalyzer chart reader, and a line drawn through these points. From this the haemoglobin value of the repeatedly sampled red cell suspension is ascertained. Since the peak height is affected by that preceding it (carry-over error) it is not sufficient to ascribe to the other whole-blood suspensions values calculated from their known dilutions, and the method of determination is as follows:-

The percentage transmission ( $T$ ) of the repeatedly sampled suspension is converted to the extinction (E) using the formula $\mathrm{E}=2-\log \mathrm{T}$. Knowing the dilutions of the suspensions, their extinctions are calculated and reconverted to percentage transmission values. These are plotted on the AutoAnalyzer chart reader against the haemoglobin values obtained by applying the dilution factors of the suspensions to the haemoglobin value ascribed to the repeatedly sampled suspension. These points give a curve obeying Beer's law, independent of the order of sampling, and a template is cut to this curve. The peaks of the red cell suspensions run in ascending order of concentration are offered up to this curve and the values read off. These values are used in the daily preparation of the calibration curve, and since they are dependent upon the order of sampling this must be invariable, i.e., in ascending order.
TEST

Each batch of blood samples for haemoglobin estimation is preceded by the four red cell suspensions. The percentage transmission value for the suspension in the $10-14 \mathrm{~g} . / 100 \mathrm{ml}$. Hb range is plotted against its ascribed haemoglobin value, and the template is used to draw a calibration curve between this point and the $99 \%$ transmission reagent baseline. The peaks of the other three suspensions are offered up to check their ascribed values, and if these are satisfactory the tests can then be read off.

\section{COMMENT}

Using the method described the results obtained with the AutoAnalyzer are generally within $0.3 \mathrm{~g} . / 100 \mathrm{ml}$. of those obtained photometrically after manual dilution. However, occasional samples will give results which agree to within $0.15 \mathrm{~g} . / 100 \mathrm{ml}$. on duplication by manual and AutoAnalyzer techniques and yet differ by up to $0.75 \mathrm{~g} . / 100 \mathrm{ml}$. when the two methods are compared.

The carry-over error with this manifold is $0.3 \mathrm{~g} . / 100 \mathrm{ml}$. when a haemoglobin value of $14.6 \mathrm{~g} . / 100 \mathrm{ml}$. is followed by a value of $3.65 \mathrm{~g} . / 100 \mathrm{ml}$., this being considerably less than that quoted in the AutoAnalyzer manual.

It is essential that all cups be covered as the fluid loss from these is $1.6 \% / \mathrm{hr}$. in still air, and may be as much as $10 \% / \mathrm{hr}$. in moving air, in which case the haemoglobin value may be increased by as much as $20 \% / \mathrm{hr}$. depending on the packed cell volume.

\section{SUMMARY}

Methods for the routine determination of haemoglobin using the AutoAnalyzer and preparation of stable red cell suspensions for the calibration of the instrument are described. 